\title{
Implementing controlled-NOT gate based on free spin qubits with semiconductor quantum-dot array
}

\author{
Yin-Zhong $\mathbf{W u}^{a}$, and Wei-Min $\mathrm{Zhang}^{b}$ \\ ${ }^{a}$ Department of Physics, Changshu Institute of Technology, Changshu 215500, P. R. China* \\ ${ }^{b}$ Center for Quantum Information Science, National Cheng Kung University, Tainan 70101, Taiwan
}

\begin{abstract}
Based on electron spins in semiconductor quantum dots as qubits, a new quantum controlledNOT(CNOT) gate is constructed in solid nanostructure without resorting to spin-spin interactions. Single electron tunneling technology and coherent quantum-dot cellular automata architecture are used to generate an ancillary charge entangled state. Using the ancillary charge entangled state as an intermediate state, we obtain a spin entangled state and design a CNOT gate by using only single spin rotations.
\end{abstract}

PACS numbers: 03.67.Lx; 73.63.-b

\section{INTRODUCTION}

The idea of using electron spins in semiconductor quantum dots as qubits [1, 2] has received great attention in the manipulation of a scalabe quantum computer. Recent experiments [3, 4], which show long spin decoherence time in semiconductor, provide a strong support for such a picture. It is well known that universal quantum computation based on electron spins can be achieved by spinspin interactions of Heisenberg type [2, 5], XY type [6, 7], and Ising type [8]. One may ask whether it is possible to implement a quantum computation scheme based on spin qubit without using spin-spin interactions. If it is possible, then such an idea can supply an alternative approach to realize quantum computation based on spin qubit. It is the main motivation of this letter. Very resently, the viewpoint of free qubit for flying fermions has been proposed [9], they construct a CNOT gate using beam splitter and single spin rotations if charge detectors are added. However, quantum computation based on free spin qubit is still lacking in solid state systems. In this letter, we shall propose an implementation of a CNOT gate based on solid state nanostructure without resorting to spin-spin interactions. We use external electrodes to control single electron tunnelings. an ancillar charge entangled state of two electrons is generated with the help of a multi-quantum-dot structure, i.e., the coherent quantum-dot cellular automata(CQCA) 10]. The charge entangled state is then converted into a spin entangled state of electrons using only single spin rotations. Spin-spin interactions are not required in our scheme, and two-qubit CNOT gate can be easily manipulated. Thus, a free spin quantum computation can be reached in semiconductor nanostructure.

\footnotetext{
*Email: yzwu@cslg.cn
}

\section{BASIC DEVICE}

Basic device in our architecture based on a semiconductor quantum-dot array is shown in Fig. 1. Three quantum dots (e.g. 1-A-B, 2-C-D) are constructed as a unit cell, and gate electrodes are integrated in each quantum dot. Solid lines in each cell indicate the possibility of interdot tunneling. The confining barrier between two cells must satisfy that the tunneling of electrons between different cells is forbidden. Moreover, there exists only one excess conductor electron in each cell. Spin states of the excess electron are chosen as qubit states. Meanwhile, four quantum dots of neighboring unit cells

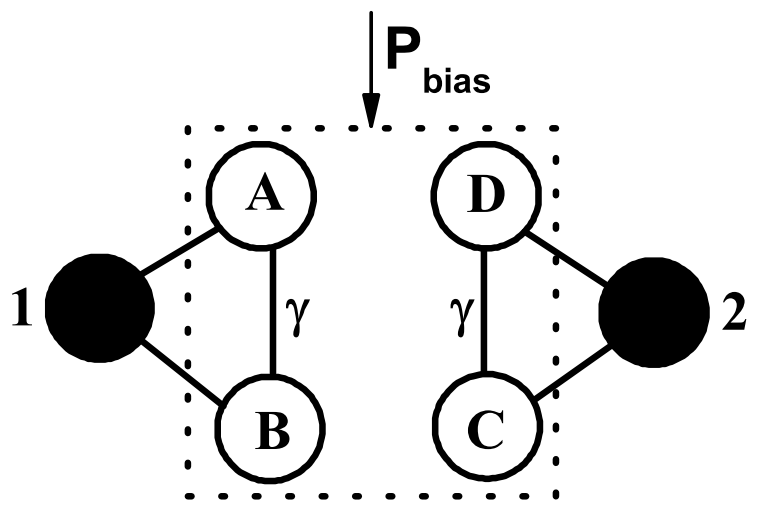

FIG. 1: Architecture of our CNOT gate composed of two unit cells(each cell contains three QDs). Four quantum dots of neighboring unit cells (e.g. ABCD) form a coherent quantumdot cellular automata structure(the dotted square box). $\gamma$ stands for the tunneling energy of electron between vertical QDs, and $P_{\text {bias }}$ denotes the bias polarization.

(e.g. ABCD) form a usual coherent quantum-dot cellular automata(CQCA)(the dotted square box in Fig. 1). In order to give a clear picture of CQCA, let's describe quantum-dot cellular automata(QCA) at first. Metal QCA has been realized in experiment to simulate the classical digital algorithm 11. A semiconductor realization of QCA structure has also been developed 12]. When the QCA structure is charged with two excess electrons, 
two electrons will occupy diagonal sites as a result of Coulomb repulsion. Moreover, only the full polarization (diagonal polarization) charge states can be existed in QCA structure. The structure of CQCA is first proposed by G. Toth 10]. Different from the charge polarization states in QCA, the charge state of two electrons in CQCA can be an arbitrary superposition state of the two full polarizations if coherent operations are performed on the full polarization states. Two input parameters $\left(\gamma\right.$ and $\left.P_{\text {bias }}\right)$ are introduced to CQCA structure [10], where $\gamma$ is the tunneling energy between vertical QDs in CQCA, $P_{\text {bias }}$ is the bias polarization which can be implemented by bias gate voltages. The effective interaction between the external bias polarization and the charge state of two electrons within CQCA can be expressed as $E_{0} P_{\text {bias }} \sigma_{z}$ [10], where $E_{0}$ is the strength of Coulombic coupling of two electrons, and the eigen states of $\sigma_{z}$ are the two full polarization states. We will show later that only a relative phase between the two full polarization states is required in our scheme. So, it is not necessary to tune the tunneling energy between vertical QDs in our CNOT gate. Turning on $P_{\text {bias }}$, the relative phase factor will be reached if $E_{0} P_{\text {bias }} \gg \gamma$. Thus, only one input parameter $P_{\text {bias }}$ in CQCA is needed to implement our CNOT gate.

\section{DEFINITION OF QUBIT STATES}

The state of the unique electron in unit cell $i$ is defined as a direct product of electron charge and spin state $\left|e_{i}\right\rangle\left|S_{i}\right\rangle(i=1,2)$. We take electron spin states as qubit states. At the initial time, the excess conductor electrons site in quantum dot 1 and 2. The excess electron in each cell is driven away from its initial position only when a two-qubit operation is performed, and electrons will be pushed back to their initial positions as soon as a two-qubit operation is completed. So, we define the electron position in unit cell $i$ at the initial time as the charge "ground state" $\left|e_{g_{i}}\right\rangle .\left|e_{1}^{A}\right\rangle$ and $\left|e_{1}^{B}\right\rangle$ are defined as the charge state of electron in the left unit siting at QDs $\mathrm{A}$ and $\mathrm{B}$, respectively, while $\left|e_{2}^{C}\right\rangle$ and $\left|e_{2}^{D}\right\rangle$ are defined as the charge state of electron in the right unit siting at QDs C and D, respectively. Control of the location of the excessive electron within each unit cell can be realized by turning on/off the gate voltage of each QD.

\section{IMPLEMENTATION OF A CNOT OPERATION}

Four steps are needed to implement a CNOT operation on the two qubits within two neighboring unit cells. Firstly, turning on the gate voltage to lower the siteenergy in $\operatorname{dots} \mathrm{A}, \mathrm{B}, \mathrm{C}$, and $\mathrm{D}$, the electron in QD 1 will tunnel into QDs $A$ and $B$. If QDs $A$ and $B$ are identical, the probabilities of electron 1 tunneling into QDs $A$ and $B$ are equal. In some sense, this process has some analogy to a flying electron moving through a beam split except that here the case is based on solid system. At the same time, electron 2 tunnels into QDs $C$ and $D$. According to the special property of cellular automata structure(Coulomb repulsion principle), two electrons will automatically occupy on the two diagonal positions in the four $\mathrm{QDs}(\mathrm{AC}$ and $\mathrm{BD})$ with equal probability. The state of the two electrons before and after this process can be written as [13]

$$
\left|e_{g_{1}}\right\rangle\left|e_{g_{2}}\right\rangle\left|S_{1}\right\rangle\left|S_{2}\right\rangle \Longrightarrow \frac{1}{\sqrt{2}}\left(\left|e_{1}^{A} e_{2}^{C}\right\rangle+\left|e_{1}^{B} e_{2}^{D}\right\rangle\right)\left|S_{1}\right\rangle\left|S_{2}\right\rangle .
$$

Then, applying bias gate voltages on electrodes in CQCA, when the duration of the bias polarization satisfies $\int_{0}^{T} E_{0} P_{\text {bias }}(t) d t=\frac{\pi}{4}$, a specific relative phase is realized, and an ancillary charge entangled state, i.e., $\frac{1}{\sqrt{2}}\left(-i\left|e_{1}^{A} e_{2}^{C}\right\rangle+\left|e_{1}^{B} e_{2}^{D}\right\rangle\right)$, can be obtained, where a global phase $e^{i \frac{\pi}{4}}$ has been discarded. As mentioned before, the strength of $E_{0} P_{\text {bias }}$ should be much larger than the tunneling energy $\gamma$. Quantum computation using two full polarization charge states in a coherent quantum-dot cellular automata as qubit states has been proposed [10]. Different from their works, here we choose electron spin states as qubit states, and the quantum-dot cellular automata is used only as an ancillary structure. So far, we did nothing upon electron spin states(qubit states). In the following steps, based on the ancillary charge entangled state, we will present how to implement a CNOT operation with only single spin rotations.

Secondly, two single spin rotations $R_{Z}^{A}(3 \pi)$ and $R_{X}^{C}(\pi)$ are performed on QDs $A$ and $C$, respectively, where $R_{a}(\theta)$ is defined as $e^{-i \theta a / 2}$. Note that the two single spin operations can be executed simultaneously. The single spin rotations can be implemented by either a local magnetic field or ultrafast optical pulses as we shall discuss later. After the second step, the state of the two excess electrons becomes

$$
\frac{1}{\sqrt{2}}\left(-i\left|e_{1}^{A} e_{2}^{C}\right\rangle R_{Z}^{A}(3 \pi)\left|S_{1}\right\rangle R_{X}^{C}(\pi)\left|S_{2}\right\rangle+\left|e_{1}^{B} e_{2}^{D}\right\rangle\left|S_{1}\right\rangle\left|S_{2}\right\rangle\right) .
$$

The third step is an inverse process of the first step. Tuning the gate voltage inversely, electron 1 and electron 2 will return their original locations, and the ancillary charge entangled state collapses into its ground state $\left|e_{1}^{A} e_{2}^{C}\right\rangle \longrightarrow\left|e_{g_{1}}\right\rangle\left|e_{g_{2}}\right\rangle,\left|e_{1}^{B} e_{2}^{D}\right\rangle \longrightarrow\left|e_{g_{1}}\right\rangle\left|e_{g_{2}}\right\rangle$. Spin entangled state is generated through the collapse of the ancillary charge entangled state, and the state of the two electrons changes to

$$
\begin{aligned}
& \left|e_{g_{1}}\right\rangle\left|e_{g_{2}}\right\rangle \frac{1}{\sqrt{2}}\left(-i R_{Z}^{A}(3 \pi)\left|S_{1}\right\rangle R_{X}^{C}(\pi)\left|S_{2}\right\rangle+\left|S_{1}\right\rangle\left|S_{2}\right\rangle\right) \\
& =\left|e_{g_{1}}\right\rangle\left|e_{g_{2}}\right\rangle e^{-i \frac{\pi}{4} \sigma_{z} \otimes \sigma_{x}}\left|S_{1}\right\rangle\left|S_{2}\right\rangle
\end{aligned}
$$

explicitly, after the third step, we write down the state of the two qubits for different initial states $|00>| 01>$, 
, $\mid 10>$, and $\mid 11>$ as following:

$$
\begin{aligned}
& \mid 00>\rightarrow \frac{1}{\sqrt{2}}(|00>-i| 01>), \\
& \mid 01>\rightarrow \frac{1}{\sqrt{2}}(-i|00>+| 01>), \\
& \mid 10>\rightarrow \frac{1}{\sqrt{2}}(|10>+i| 11>), \\
& \mid 11>\rightarrow \frac{1}{\sqrt{2}}(i|10>+| 11>) .
\end{aligned}
$$

Fourthly, we do another two single qubit rotations $R_{Z}^{1}(3 \pi / 2)$ and $R_{X}^{2}(3 \pi / 2)$ on QDs 1 and 2 , respectively. We note that the two single spin operations can also be performed at the initial time. When the four steps are completed, we get a CNOT operation acting on two qubits within the basic device, namely,

$$
\begin{aligned}
& |00>\rightarrow| 00>, \\
& |01>\rightarrow| 01>, \\
& |10>\rightarrow| 11>, \\
& |11>\rightarrow| 10>.
\end{aligned}
$$

In our scheme, single qubit rotation can be realized via a local magnetic field or ultrafast laser pulses. Single spin rotations $R_{X}$ and $R_{Z}$ can be implemented by turning on local magnetic fields along $x$ direction and $z$ direction, respectively. However, one-qubit operation by applying a magnetic field is very slow. For speeding up the operations, we shall use two laser pulses to generate a single spin rotation through adiabatic process 14. or use optical "tipping" pulse 15]. In the following, we will describe how to realize a single spin rotation by using Raman transition with adiabatic process. Two pulsed lasers with different polarizations are addressed on a quantum dot. For a large detuning and under Raman resonance $\left(\Delta=\Delta_{1}=\Delta_{2} \gg \Omega_{e f f}\right)$, i.e., adiabatically eliminating the excited state 14], an effective interaction between spin-up state $|0\rangle$ and spin down state $|1\rangle$ can be realized: $\Omega_{e f f}(t)\left(|0\rangle\langle 1| e^{-i\left[\left(\omega_{1}-\omega_{2}\right) t+\delta \phi_{12}\right]}+\right.$ $\left.|1\rangle\langle 0| e^{i\left[\left(\omega_{1}-\omega_{2}\right) t+\delta \phi_{12}\right]}\right)$, where $\Omega_{e f f}(t)=\frac{\Omega_{1}(t) \Omega_{2}(t)}{\Delta}, \delta \phi_{12}$ is the initial phase difference between the two lasers, $\Delta_{1}$ and $\Delta_{2}$ denote the detuning of two lasers, respectively, and $\Omega_{1}\left(\Omega_{2}\right)$ denotes Rabi frequency between spinup state $|0\rangle$ (spin-down state $|1\rangle)$ and the excited state of the quantum dot. By properly adjusting the duration and the initial phase of each laser pulse to satisfy $\int_{0}^{T} \Omega_{e f f}(t) d t=\frac{\theta}{2}$ and $\left(\omega_{1}-\omega_{2}\right) T+\delta \phi_{12}=2 n \pi$, a single spin rotation $R_{X}(\theta)$ is achieved, and $R_{Y}(\theta)$ could be implemented under the conditions $\int_{0}^{T} \Omega_{\text {eff }}(t) d t=\frac{\theta}{2}$ and $\left(\omega_{1}-\omega_{2}\right) T+\delta \phi_{12}=2 n \pi+\frac{3 \pi}{2}$. Combining $R_{X}(\theta)$ with $R_{Y}(\theta)$, we can obtain an arbitrary single spin rotation $R_{Z}(\theta)$. For example, single spin rotation $R_{Z}(3 \pi)$ performed on QD $A$ can be achieved by $R_{X}(3 \pi) R_{Y}(\pi)$, and single spin rotation $R_{Z}\left(\frac{3 \pi}{2}\right)$ performed on QD 1 can be realized by three series operations $R_{Y}\left(\frac{3 \pi}{2}\right) R_{X}\left(\frac{\pi}{2}\right) R_{Y}\left(\frac{\pi}{2}\right)$ - In principle, QDs 1, 2, $A$ and $C$ can be different in size. Masks might be manufactured upon QDs $B$ and $D$ [16], then the laser pulse can selectively couple to an individual QD, and the laser field need not be localized spatially in our scheme.

As the initial state preparing is concerned, bias gate voltages can be used to lower the on-site energy of dots 1 and 2 for the initialization of charge state, and a static uniform magnetic field can be applied to split spin-up state and spin-down state by the Zeeman energy for the initialization of spin state(qubit state).

Recently, single electron tunneling through triple virtual quantum dots has been studied in experiment [17]. Although our scheme would be a challenge in technology, it gives a new way on how to implement free spin quantum computation based on solid QDs array. Such structures could be fabricated in scalable, and we envisage that the separation between each CNOT gate structure would be greater than the size of them. This ensure that the Coulomb repulsion between electrons in different CNOT gates is always smaller than the Coulomb repulsion between two electrons within the same cellular automata structure and may be treated as perturbation. As decoherence time and operation time are concerned, the typical decoherence time of electron spin in GaAs $\mathrm{QD}$ is $50 \mu \mathrm{s}[4]$, the operation time of single spin rotation by ultrafast pulse is several picoseconds, and the time of single electron tunneling between two coupled QDs is about a few $100 \mathrm{ps}[18]$. Thus, the great challenge in our scheme might be the fast control technology of SET [19]. Here, We focus our attention on how to design a CNOT gate, the architecture for scalable quantum computation, measurement, and how to use this basic device to produce spin Bell states will be discussed elsewhere 20]. We hope the device can be fabricated by current or future nanotechnology, and the CNOT gate might be tested firstly in experiment before a scalable quantum computation is put forward.

\section{CONCLUSION}

In summary, an ancillary charge entangled state is generated based on semiconductor coherent quantum-dot cellular automata. Since spin and charge are commute, the spin entangled state is realized based on the ancillary charge entangled state. A CNOT gate based spin qubits is first implemented in solid system without using of qubit-qubit interactions. Combining with single qubit operations, a scalable free spin quantum computation could be realized in semiconductor nanostructure. 
[1] M. A. Nielsen, and I. L. Chuang, Quantum Computation and Quantum Information, (Cambridge University Press, 2000).

[2] D. Loss, and D. P. DiVincenzo, Phys. Rev. A 57, 120 (1998).

[3] D. D. Awschalom, D. Loss, and N. Samarth, Semiconductor Spintronics and Quantum Computation, (SpringerVerlag Berlin Heidelberg, 2002).

[4] Rogerio de Sousa, and S. Das Sarma, Phys. Rev. B 67, 033301(2003); B. Beschoten, E. Johnstonhalperin, D. K. Young, M. Poggio, J. E. Grimaldi, S.Keller, S. P. DenBaars, U. K. Mishra, E. L. Hu, and D. D. Awschalom, Phys. Rev. B 63, 121202(R)(2001).

[5] D. P. DiVincenzo, D. Bacon, J. Kempe, G. Burkard, and K. B. Whaley, Nature 408, 339 (2000).

[6] D. A. Lidar, and L. A. Wu, Phys. Rev. Lett. 88, 017905 (2002).

[7] A. Imamoglu, D. D. Awschalom, G. Burkard, D. P. DiVincenzo, D. Loss, M. Sherwin, and A. Small, Phys. Rev. Lett. 83, 4204(1999).

[8] Jonathan A. Jones, Phys. Rev. A67, 012317(2003).

[9] C. W. J. Beenakker, D. P. DiVincenzo, C. Emary, and M. Kindermann, Phys. Rev. Lett. 93, 020501(2004).

[10] Geza Toth, and Craig S. Lent, Phys. Rev. A 63, 052315(2001).

[11] Islamshah Amlani, Alexei O. Orlov, Geza Toth, Gary H. Bernstein, Craig S. Lent, and Gregory L. Snider, Science 284, 289(1999).

[12] Y. Fu, and M. Willander, J. Appl. Phys. 83, 3186(1998);
M. Govemale, M. Macucci, G. lannaccone, and C. Ungarelli, J. Appl. Phys. 85, 2962(1999); M. Macucci, M. Gattobigio, and G. Iannaccone, J. Appl. Phys. 90, 6428(2001).

[13] As described by Eq. (6) in Ref. [10], when two parameters $\gamma$ and $P_{\text {bias }}$ are introduced into the QCA structure, one can obtain an arbitrary superposition state of the two diagonal polarization states. One can also abtain Eq. (1) by employing the following two steps. Firstly, two electrons in two unit cells are driven to one of the diagonal polarization state. Secondly, by applying coherent operations $\left(\sigma_{X}, \sigma_{Z}\right)$ on the diagonal polarization state, the charge entangled state, described by Eq. (1), can be realized.

[14] Nikolay V Vitanov, Thomas Halfmann, Bruce W Shore, and Klaas Bergmann, Annu. Rev. Phys. Chem. 52, $763(2001)$.

[15] J. A. Gupta, R. Knobel, N. Samarth, D. D. Awschalom, Science 292, 2458 (2001).

[16] A. Zrenner, E. Beham, S. Stuffer, F. Findels, M. Bichler, and G. Abstrelter, Nature 418, 612 (2002).

[17] A. Vidan, R. M. Westervelt, M. Stopa, M. Hanson, and A. C. Gossard, Appl. Phys. Lett 85, 3602(2004).

[18] T. Hayashi, T. Fujisawa, H. D. Cheong, Y. H. Jeong, and Y. Hirayama, Phys. Rev. Lett.91, 226804 (2003).

[19] H. Grabert, and M. H. Devoret, Single charge tunneling:coulomb blockade phenomena in nanostructures, (Plenum Press, New York, 1992).

[20] W. M. Zhang, Y. Z. Wu, and Chopin Soo(submitted). 